\title{
ANÁLISIS DE CORROSIÓN EN DUCTOS DE CONDUCCIÓN DE GASES EN UNA PLANTA DE CLINKER
}

\section{CORROSION ANALYSIS IN GAS CONDUCTION DUCTS IN A CLINKER PLANT}

\author{
Alex Humberto Restrepo Carvajal ${ }^{1} \quad$ Félix Echeverría Echeverría $^{1}$ \\ Recibido 13 de junio de 2008, aceptado 9 de octubre de 2008 \\ Received: June 13, 2008 Accepted: October 9, 2008
}

\begin{abstract}
RESUMEN
En este trabajo se presentan los resultados de un estudio detallado de las causas del deterioro observado en ductos metálicos de una planta productora de cemento. Para el análisis, se inició con una minuciosa inspección visual, tomando muestras de sustratos, productos de corrosión y gases de proceso. Al mismo tiempo se realizaron medidas de potencial de estructura, espesores de pared y temperaturas de superficies. Para la evaluación de la agresividad del ambiente se instalaron captadores de contaminantes (cloruros, sulfatos y óxidos de nitrógeno). Las muestras recolectadas se analizaron por medio de microscopia óptica y electrónica de barrido, espectroscopia EDS, difracción de rayos X, espectroscopia infrarroja y cromatografía iónica. Los gases se analizaron in situ siguiendo el procedimiento EPA-2. Los resultados para las superficies externas indican una atmósfera con agresividad C3 según la norma ISO 9223, observando influencia de la alta temperatura, largos periodos de humectación, material particulado y errores en la selección del sistema de protección. Las capas de productos al exterior son uniformes, compactas y son compuestas principalmente por hematita. Al interior de los ductos se presentan procesos de sulfurización y oxidación; el ataque por azufre es localizado y genera perforación de las paredes metálicas. Los productos encontrados son mayormente sulfatos de hierro y $\mathrm{Fe}_{2} \mathrm{O}_{3}$. Los procedimientos empleados y los resultados obtenidos son útiles para la toma de decisiones en mantenimiento y protección contra la corrosión de este tipo de instalaciones.
\end{abstract}

Palabras clave: Oxidación, corrosión atmosférica, corrosión a alta temperatura, evaluación de corrosividad.

\begin{abstract}
The results of a detailed study of the causes of deterioration, observed in metallic ducts in a cement production plant are presented. The analysis started with a careful visual inspection, collecting samples of substrates, corrosion products and process gases. At the same time, measurements of potential, wall thickness and surface temperatures were carried out. For environment aggressiveness evaluation, pollutant (chlorides, sulfates and nitrogen oxides) collectors were installed. The collected samples were analyzed by optic and scanning electron microscopy, EDS spectroscopy, X ray diffraction, infrared spectroscopy and ionic chromatography. Gases were analyzed in-situ following EPA-2 procedure. The results for the external surfaces indicate an atmospheric aggressiveness C3 according to ISO 9223 standard, showing the influence of high temperature, long humectation periods, particulate matter and mistakes in the selection of the protection system. The products layers at the exterior are uniform, compact and are mainly composed of hematite. Sulfuration and oxidation processes are found inside the ducts; the sulfur attack is localized and generates perforation of the metallic walls. The products found are mostly iron sulfates and $\mathrm{Fe}_{2} \mathrm{O}_{3}$. The procedures employed and the results obtained are useful for decision making on maintenance and corrosion protection of this kind of installations.
\end{abstract}

Keywords: Oxidation, atmospheric corrosion, high temperature corrosion, corrosivity evaluation.

\footnotetext{
1 Grupo de Corrosión y Protección, SIU. Universidad de Antioquia. Calle 62 No. 52-59. Medellín, Colombia. E-mail: oahrc797@udea.edu.co, fecheve@udea.edu.co
} 


\section{INTRODUCCIÓN}

En los últimos años en una planta productiva de cemento se ha presentado un problema de corrosión tanto en las chimeneas de los hornos de clinker, como en varias de las estructuras de conducción de gases. Este problema se ha agravado paulatinamente generando mayores labores de mantenimiento y los métodos de protección aplicados hasta hoy no han mostrado resultados satisfactorios para prevenir dicho deterioro. En el proceso de producción de cemento blanco se utilizan como materias primas los siguientes minerales: caliza, arena silícea, fluorita y caolín. Todas ellas en proporciones apropiadas para generar el crudo, el cual se procesa por vía húmeda y con ella se alimentan los hornos de tipo rotatorio. La adición de fluorita es un proceso relativamente reciente (últimos 10 años) y fue implementado con el objeto de fomentar la formación tanto de silicato tricálcico como de silicato bicálcico a menores temperaturas de trabajo y además aumentar la resistencia a la compresión.

En el proceso de combustión inicialmente se empleaba petróleo crudo como combustible de los hornos, pero debido a los altos costos, en los últimos 10 años se cambió al uso de carbón mineral. Análisis de los carbones empleados indican entre otros valores promedio $11,7 \%$ de cenizas, $30,7 \%$ de material volátil y $0,6 \%$ de azufre, considerado apropiado para el proceso. Diariamente se hace un estricto control tanto de las materias primas, como del producto obtenido. En la actualidad, esta planta opera cuatro hornos rotatorios para la producción de clinker y uno para la producción de cal. Las temperaturas de los procesos van desde aproximadamente 400 hasta $1.350{ }^{\circ} \mathrm{C}$. Sensores internos controlan porcentajes de oxígeno, cantidad de combustible y temperaturas.

El material de fabricación de los ductos de aire, así como de la chimenea, es acero al carbono A-36 de aproximadamente $5 \mathrm{~mm}$ de espesor; acero AISISAE 1010, también es empleado en algunas partes. Pinturas especialmente resistentes a la temperatura han sido empleadas para las actividades de mantenimiento; en unas zonas se ha empleado una pintura de aluminio que resiste entre $260-560^{\circ} \mathrm{C}$. El espesor de la lámina se monitorea por medio de medidas de ultrasonido, así para el horno de cal se tienen reducciones de alrededor del $60 \%$ del espesor original. La planta se encuentra en una región de clima templado con oscilaciones de humedad relativa entre 45 y $83 \%$, temperaturas en el rango de 25 y $40{ }^{\circ} \mathrm{C}$ y una alta pluviosidad. Los gases de descarga a la atmósfera continuamente se muestrean con respecto a contenidos de partículas, óxidos de azufre, óxidos de nitrógeno, $\mathrm{CO}, \mathrm{CO}_{2}$ y oxígeno.

\section{PARTE EXPERIMENTAL}

La metodología seguida para este caso constó primero de una detallada inspección visual de la planta, durante la cual se tomaron muestras de substratos, productos de corrosión $\mathrm{y}$ gases del proceso, al igual que se realizaron mediciones del potencial de la estructura, espesores, temperatura de superficie y corrosividad del microclima. Para los análisis de las muestras de substratos y productos de corrosión se empleó microscopia óptica y electrónica de barrido (SEM), junto con espectroscopia de energía dispersiva (EDS), difracción de rayos X (DRX), espectroscopia infrarroja con transformada de Fourier (FTIR) y cromatografía iónica (IC). Los gases fueron analizados siguiendo el procedimiento establecido en el método EPA-2. La corrosividad se determinó cuantificando los contenidos en la atmósfera respecto a cloruros (norma NBR 6211), sulfatos (norma NBR 6921) y óxidos de nitrógeno [1]. Se instalaron tres estaciones de muestreo conteniendo captadores para cada especie. Para la medición de temperatura se empleó un sistema infrarrojo y para los espesores un medidor de ultrasonido. Los difractogramas de rayos $\mathrm{X}$ se obtuvieron empleando una fuente de cobre y se analizaron con el programa PHASAN X. Los espectros infrarrojos se obtuvieron en un espectrómetro Nicolet Avator 330 FT-IR con reflectancia difusa, analizando el rango de 400 a $4.000 \mathrm{~cm}^{-1}$.

Un microscopio electrónico JMS-5910 JEOL con analizador EDS fue usado para observación morfológica y análisis elemental. En análisis de cromatografía iónica se realizó en un cromatógrafo Compact IC 761 Metrohm y las muestras se prepararon lixiviando las especies solubles contenidas en los productos en agua ultrapura por dos horas. Las mediciones del potencial se llevaron a cabo mediante la utilización de un electrodo de calomel y haciendo el contacto con la estructura por medio de una esponja humectada en $\mathrm{KCl}$.

\section{RESULTADOS Y DISCUSIÓN}

\section{Análisis por inspección visual}

Se pudo observar corrosión generalizada en las estructuras que componen los sistemas de ductos y chimeneas (ver figura 1). El fenómeno se encuentra asociado a superficies expuestas a mediana y alta temperatura, que no cuentan con protección externa adecuada. En la mayoría de los casos el sistema de pintura falló, exponiendo la superficie a la interacción con la atmósfera; en algunos casos se observó el recubrimiento en buenas condiciones, lo cual se pudo explicar por ser de mayores especificaciones que en las superficies deterioradas. La falta de correctas especificaciones se pudo observar claramente en la 
chimenea donde el área donde la temperatura estaba por debajo de $70^{\circ} \mathrm{C}$ presentaba una película de pintura en buen estado mientras arriba de esta, donde los gases elevan la temperatura del material, el sistema de pintura no presentó buen desempeño (ver figura 2). En algunas partes el ataque es tan avanzado que se han perdido paneles completos (ver figura 3). En general se observa que uniones y partes soldadas presentan mayor deterioro.

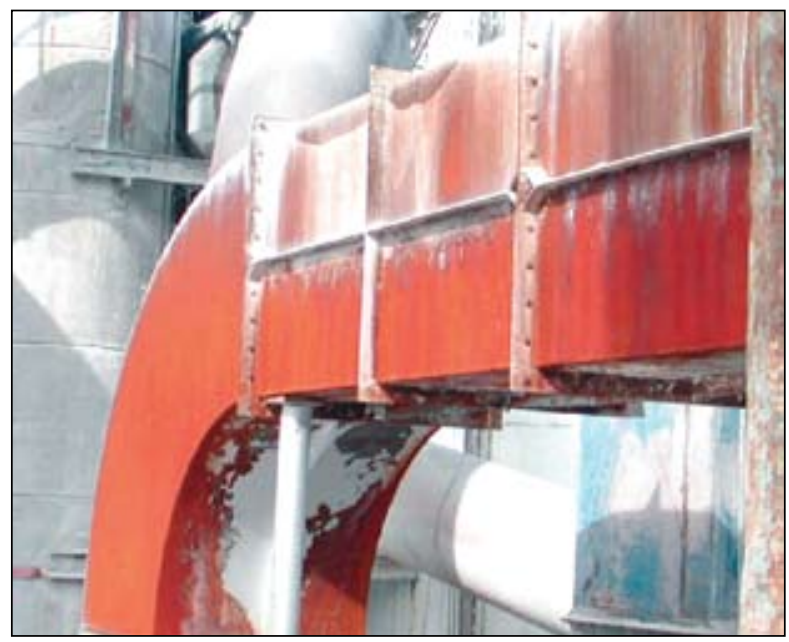

Figura 1. Corrosión generalizada en un ducto de aire de la planta de clinker.

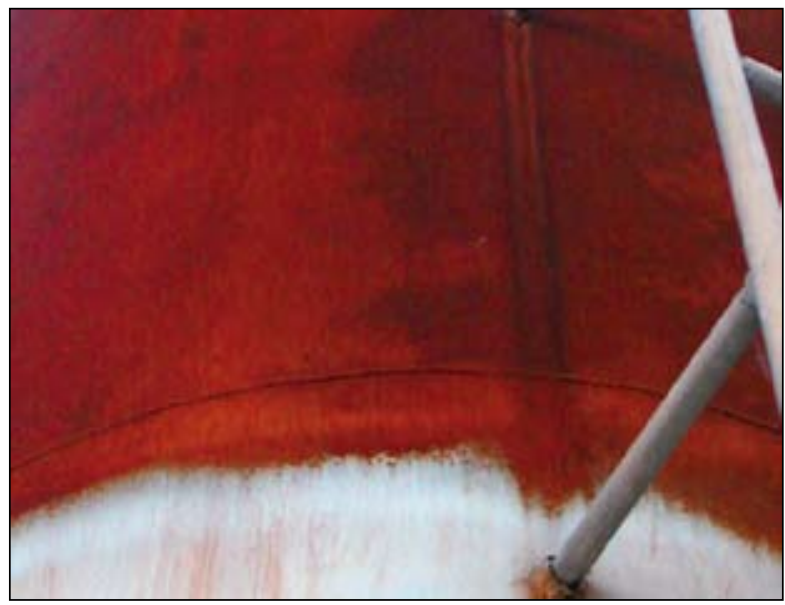

Figura 2. Detalle de la base de la chimenea de la planta de clinker, mostrando zonas con diferente estado superficial en la misma estructura.

\section{Análisis de los productos de corrosión encontrados}

Se analizaron muestras de productos tomadas en cuatro diferentes partes de la planta, una del interior de uno de los hornos y las otras tres de superficies externas. En la figura 4 se presentan los espectros IR obtenidos para cada muestra.
El espectro de la figura $4 \mathrm{a}$, correspondiente a productos recogidos en el interior de un horno, muestra bandas fuertes en aproximadamente $1168,1128,669 \mathrm{~cm}^{-1}$ y débiles alrededor de 1627 y 2127 , las cuales revelan la presencia de sulfato de calcio $\left(\mathrm{CaSO}_{4}\right)$ [2], que es un compuesto derivado del proceso de clinquerización y formado por la reacción de los gases de combustión con las materias primas.

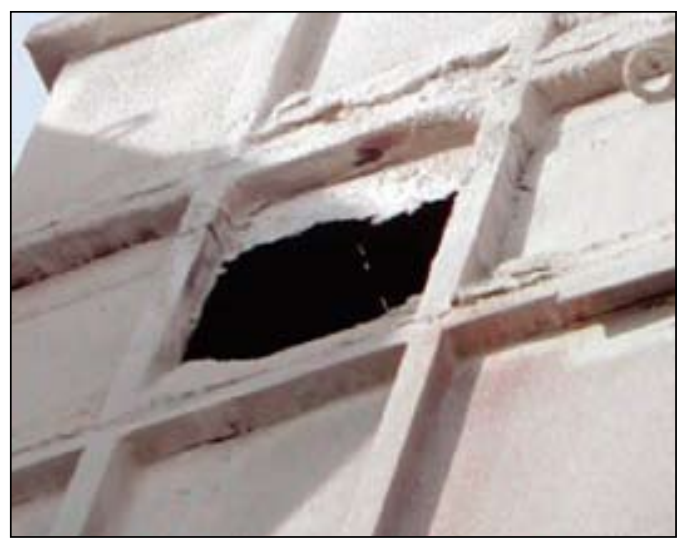

Figura 3. Detalle de un ducto de aire de un horno de clinker, mostrando la pérdida de un panel metálico debido a ataque por corrosión.
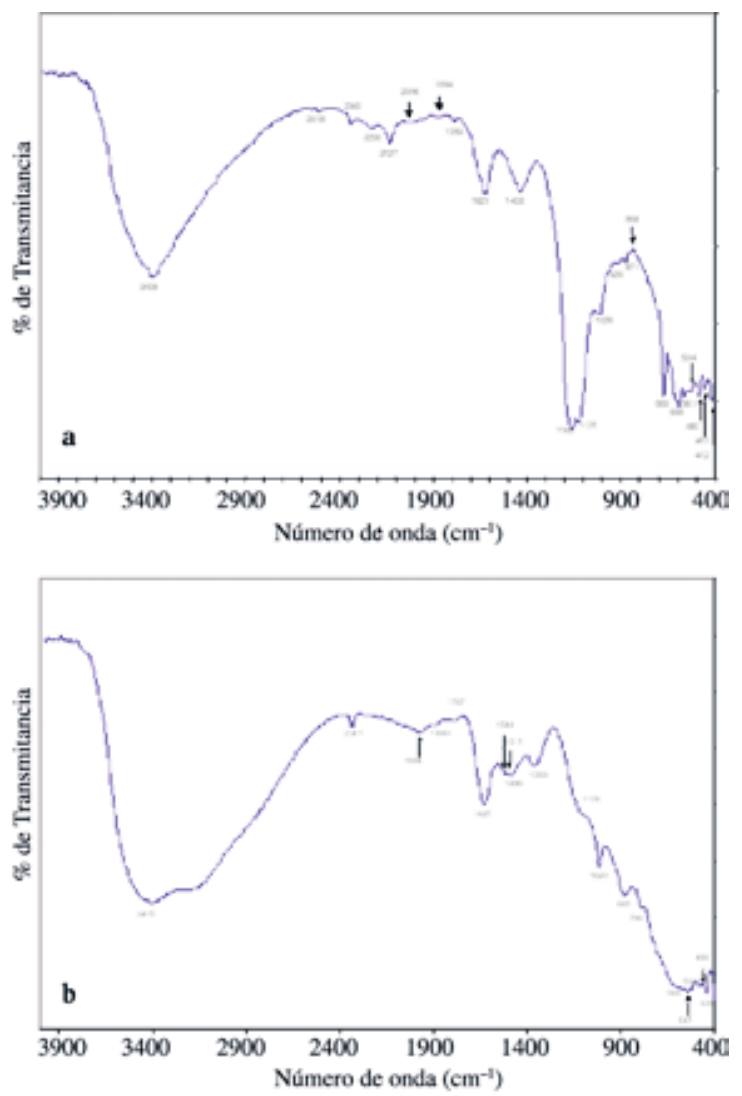

Figura 4. Espectros de infrarrojo: a) Interior de horno, b) chimenea lado externo. 
También se observan bandas cercanas a 1.438 y $871 \mathrm{~cm}^{-1}$ lo cual es característico de carbonato de calcio $\left(\mathrm{CaCO}_{3}\right)$, una de las materias primas en el proceso. De otro lado, se evidencia la presencia de óxidos de hierro de tipo $\mathrm{Fe}_{2} \mathrm{O}_{3}$ más específicamente Maghemita con bandas a 561, 480 y $412 \mathrm{~cm}^{-1}$ y la forma del espectro en el rango entre 588 y $1.128 \mathrm{~cm}^{-1}$ hacen suponer un compuesto de tipo sulfato de hierro hidratado; una posible composición es dada por la fórmula $\mathrm{NaFe}_{3}\left(\mathrm{SO}_{4}\right)_{2}(\mathrm{OH})_{6}$ [2].

En la figura $4 \mathrm{~b}$, el espectro tomado a productos provenientes de la superficie externa de la chimenea muestra bandas características del hidróxido de hierro conocido como Lepidocrocita $\left(1020\right.$ y $\left.885 \mathrm{~cm}^{-1}\right)$, además de un compuesto oxidado tipo $\mathrm{Fe}_{2} \mathrm{O}_{3}\left(400-500 \mathrm{~cm}^{-1}\right)$ [3]. La presencia de un oxihidroxido de hierro a las condiciones de temperatura arriba de los $100{ }^{\circ} \mathrm{C}$ es algo inesperada; sin embargo, teniendo en cuenta que la superficie metálica está expuesta a la intemperie, dentro de los varios mecanismos para la formación de óxidos se podría considerar la deshidroxilación o pérdida de $\mathrm{OH}^{-}$de la lepidocrocita [4]. Adicional a lo anterior este espectro también revela presencia de sulfato de y carbonato de calcio, aunque en mucho menor cantidad que la muestra del interior, como lo sugiere la poca intensidad de los picos correspondientes. Los espectros de otras muestras de productos tomados de superficies externas de ductos de aire revelan composición de los productos similar a la chimenea. Sin embargo, se observó un aumento en la intensidad de los picos correspondientes a sulfato y carbonato de calcio y ocurrencia de picos que sugieren la presencia de hematita, lo cual es confirmado por los resultados de DRX presentados a continuación. Los compuestos de calcio seguramente resultan de la deposición de material particulado suspendido en la atmósfera de la planta.

El compuesto predominante en la muestra proveniente del interior (figura 5a) es el sulfato de calcio cristalino (anhydrita), con menores cantidades de hematita y calcita. La hematita es producto común de los fenómenos de corrosión a alta temperatura del acero e igualmente en este caso fue encontrada como componente principal en las muestras de productos tomados de superficies externas. La calcita es una de las materias primas del proceso. Además de hematita, los productos encontrados en las superficies externas contienen otras especies oxidadas de hierro como goethita y magnetita (ver figura 5b). La goethita es seguramente resultado de la interacción de la superficie con la humedad ambiental, mientras la magnetita resulta de procesos prolongados de oxidación [4]. De otro lado, pequeñas cantidades de sílice y carbonato de calcio fueron observadas en las muestras tomadas de superficies externas; éstas seguramente resultan de la deposición de material particulado de la atmósfera.
Análisis metalográfico de varias muestras de material tomadas de los diferentes partes bajo estudio, indican una microestructura ferrítico-perlítica, con una ferrita equiaxial y un tamaño de grano entre 8 y 9 . El porcentaje de perlita es del orden del $20 \%$ (figura 6).
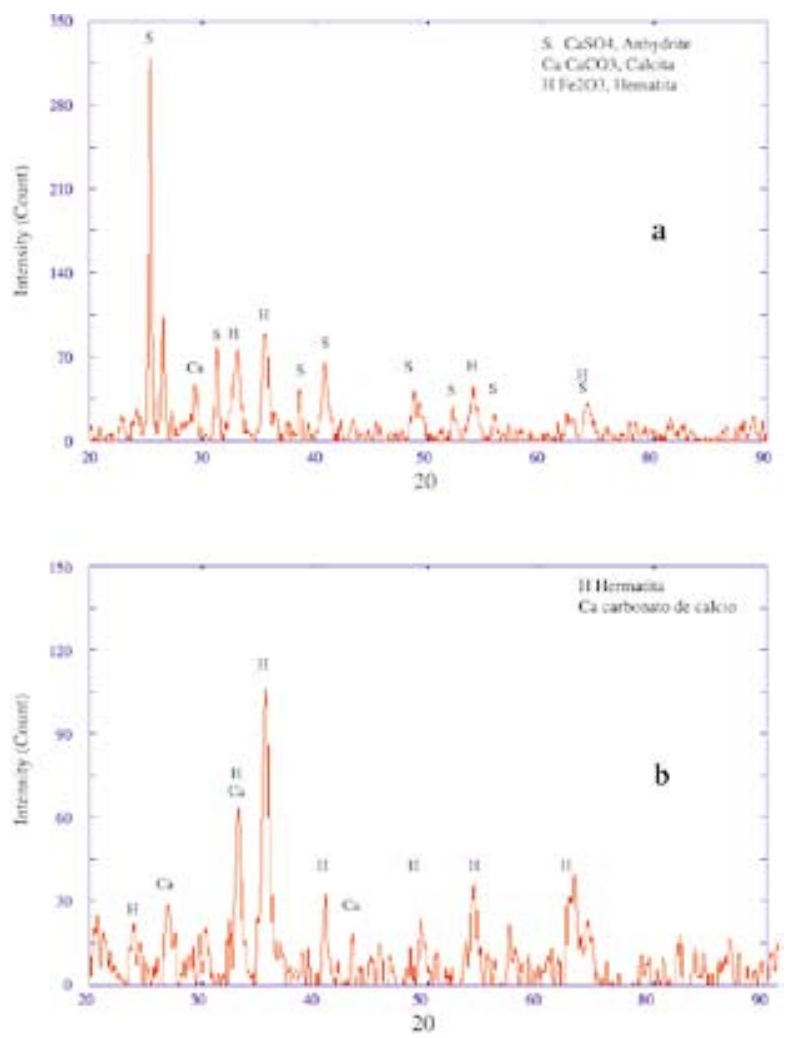

Figura 5. Difractogramas de muestras provenientes de: a) interior del horno, b) chimenea lado externo.

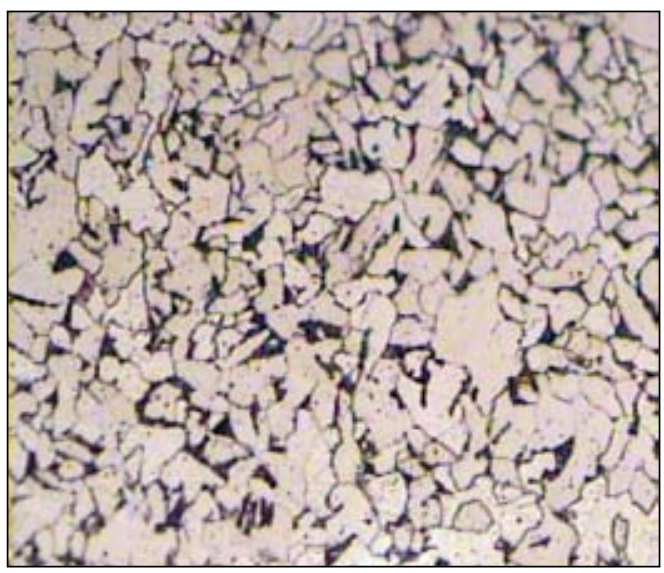

Figura 6. Metalografía de la lámina tomada de los ductos de aire (50X). 

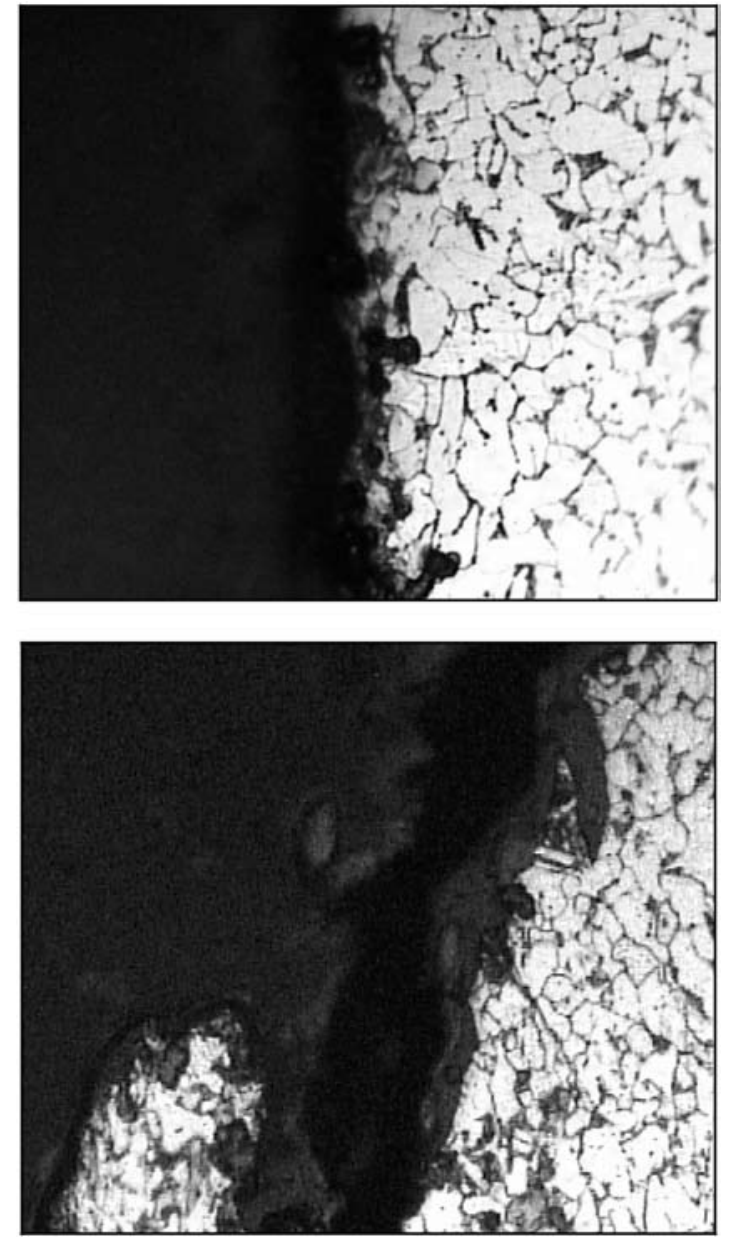

Figura 7. Micrografías de muestras en corte transversal de superficies externas (arriba) e internas (abajo) (50X).

Asimismo se prepararon muestras en corte transversal para analizar la morfología del ataque tanto en superficies interiores como exteriores (figura 7). La superficie del lado externo expuesta a corrosión atmosférica presenta un ataque uniforme y poca penetración en el sustrato. La morfología del ataque por el lado interno, correspondiente a la superficie expuesta al efecto de los gases de combustión, indica un ataque más intenso, penetrando considerablemente en el sustrato e incluso llegando a perforar la pared en su totalidad. En la micrografía de esta muestra se observa cómo el ataque interno alcanza a desprender porciones del metal base y genera ataque por picado que drásticamente aceleran el ataque corrosivo. Los productos de corrosión en general están cubiertos por una capa blanca derivada del proceso de producción del clinker que al parecer no genera ningún tipo de protección sobre la lámina metálica. Según los resultados de DRX y FTIR este material corresponde principalmente a sulfato de calcio con bajos contenidos de carbonato de calcio. Estos depósitos enriquecidos en $\mathrm{CaSO}_{4}$, al parecer intervienen en el ataque del metal modificando el medio en el cual está expuesto el acero, al disminuir la presión parcial de oxígeno e incrementar aquella del sulfuro [5].

Análisis de las mismas interfaces mediante SEM revelan para el caso de la superficie externa una película de óxido de carácter compacto y de aparente uniforme composición sobre el substrato (figura 8), mientras que para la superficie expuesta a los gases de combustión en el interior (figura 9), se observan capas de óxido y de carbonato entremezcladas y de mayor espesor, en este caso el espesor total es mayor a $200 \mu \mathrm{m}$, mientras que para la superficie expuesta a la atmósfera no son mayores a $70 \mu \mathrm{m}$.
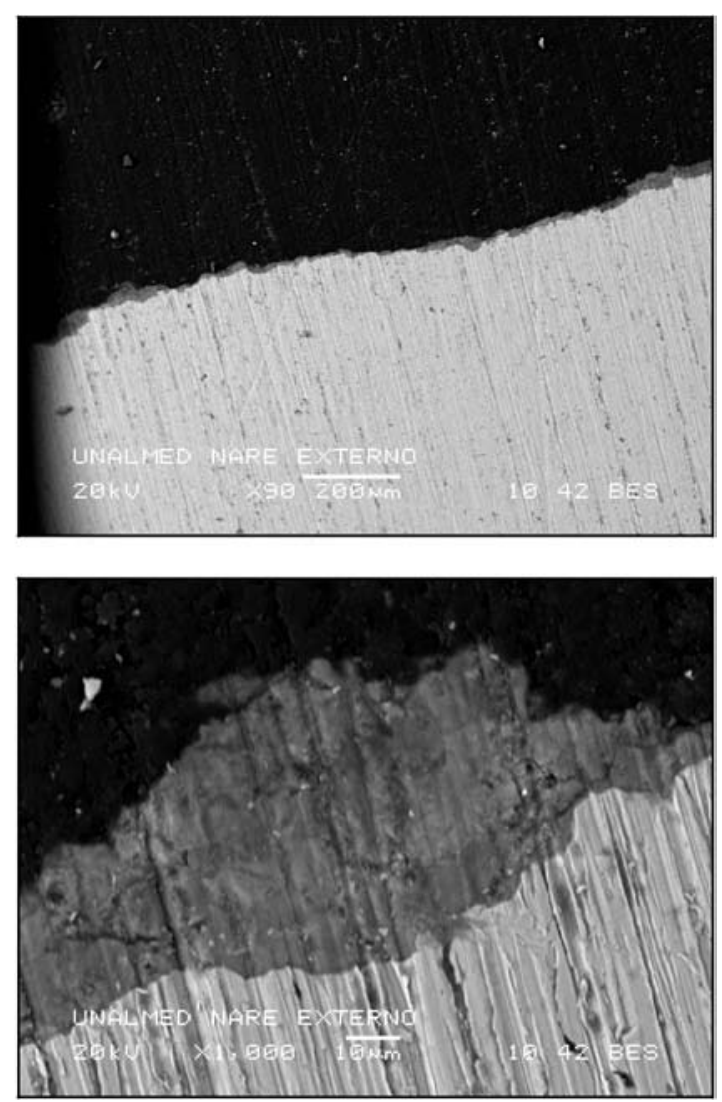

Figura 8. Micrografías SEM de la superficie externa de la lámina (90X arriba - 1000X abajo).

El análisis EDS de la película de óxido formada en la superficie externa revela, además de $\mathrm{Fe}(\sim 26 \%$ at.) y $\mathrm{O}$ ( $40 \%$ at.), un porcentaje relativamente bajo de calcio ( $\sim 1 \%$ at.). Trazas (<1\% at.) de manganeso y cloro también fueron detectadas; lo primero proviene del metal base mientras que el cloro es seguramente un contaminante atmosférico. La composición de los productos formados en la superficie interna fueron igualmente analizados por EDS en dos zonas: capa de óxido cerca de la interfase con 
el metal base y en la capa de carbonato. Similar a la capa de óxido formada en la superficie exterior los componentes principales son $\mathrm{Fe}$ y $\mathrm{O}$ con pequeñas cantidades de $\mathrm{Ca}$, $\mathrm{S}$ y Si. En la otra zona analizada $\mathrm{Ca}(\sim 19 \%$ at. $), \mathrm{O}$ (56\% at) y S ( $~ 8 \%$ at.) son los componentes principales conteniendo porcentajes menores de $\mathrm{Al}, \mathrm{Si}$ y $\mathrm{Cl}$. Los anteriores resultados en su mayoría coinciden con los resultados de FTIR y DRX, evidenciando especies oxidadas de hierro, con presencia de carbonato y sulfato de calcio. Dado su importante grado de agresividad debe resaltarse la presencia de $\mathrm{Cl}$ aunque se encuentra en pequeñas cantidades; los resultados de cromatografía iónica más abajo también evidencian su existencia haciendo parte de los productos.
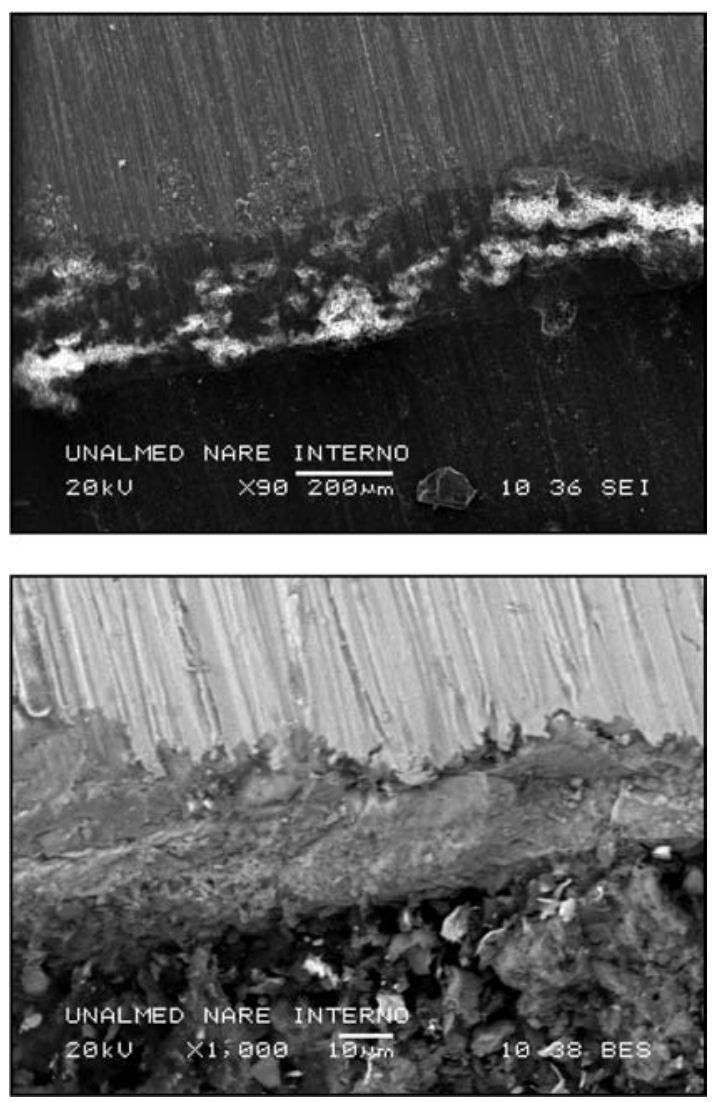

Figura 9. Micrografías SEM de la superficie interna de la lámina (90X arriba - 1000X abajo).

Por cromatografía iónica se analizaron los contenidos de las especies agresivas en las muestras de productos recolectados. Según estos análisis para la muestra tomada de la superficie interna se encontraron unos contenidos aproximados de $25,7 \mathrm{~g} \mathrm{~m}^{-2}$ de sulfatos, $0,7 \mathrm{~g} \mathrm{~m}^{-2}$ de cloruros y $0,3 \mathrm{~g} \mathrm{~m}^{-2}$ de óxidos de nitrógeno. Para el lado externo $1,2 \mathrm{~g} \mathrm{~m}^{-2}$ de sulfato y $0,2 \mathrm{~g} \mathrm{~m}^{-2}$ de cloruros. Como se observa, los contenidos de contaminantes varían ampliamente dependiendo si es la superficie interna o externa la analizada. Los contenidos menores encontrados en las muestras de la superficie externa se pueden explicar debido al efecto de las lluvias, disolviendo y lavando la superficie. A pesar de esto, los contenidos de sulfatos medidos en los productos de la superficie externa inducirán la falla del sistema de pintura si consideramos el umbral máximo de aproximadamente $0,596 \mathrm{~g} \mathrm{~m}^{-2}$ [6]. Los altos contenidos de sulfatos en el interior están claramente relacionados con la existencia de sulfato de calcio, como indicaron los resultados de FTIR y DRX. Este compuesto puede actuar como fuente de iones sulfato que aceleran el ataque de la superficie. Para explicar la presencia de cloruros se analizó una muestra de pasta tomada a la entrada del horno de clinker. Los resultados de este ensayo claramente mostraron que estos iones provienen de la pasta de proceso, con contenidos de alrededor de $1371 \mathrm{mg} \mathrm{L}^{-1}$ para cloruros, $22 \mathrm{mg} \mathrm{L}^{-1}$ para fluoruros, $12 \mathrm{mg} \mathrm{L}^{-1}$ para nitratos y $9 \mathrm{mg} \mathrm{L}^{-1}$ para sulfatos. Las bajas cantidades de sulfatos lixiviados en la pasta indican que estos compuestos son generados durante el proceso de combustión y muy posiblemente provienen del azufre contenido en el carbón empleado como combustible. La presencia de fluoruros es seguramente originada en la fluorita intencionalmente adicionada a la materia prima.

Las medidas del potencial de la estructura en dos áreas diferentes arrojan valores promedio de $-0,176 \mathrm{~V}$ vs. EEH (electrodo estándar de hidrógeno) y -0,034 V vs. EEH. Para que una estructura se encuentre protegida, su potencial medido con respecto al electrodo de hidrógeno debe estar por debajo de $-0,65 \mathrm{~V}[7,8]$; así los valores medidos indican que las superficies se encuentran en estado activo, es decir el proceso de corrosión continúa deteriorando la estructura.

Las mediciones de temperatura externa buscaban establecer los rangos habituales de temperatura de trabajo en los equipos. Para los hornos de clinker se tienen variaciones de temperaturas en las superficies metálicas entre 193 y $108^{\circ} \mathrm{C}$, dependiendo la distancia recorrida por los gases de combustión en el ducto, a medida que se alejan del horno disminuyen temperatura. Para el horno de cal la temperatura está entre de 97 y $90{ }^{\circ} \mathrm{C}$. Análisis de los registros del cuarto de control sin embargo indican que se tienen aumentos bruscos de temperatura de hasta $270{ }^{\circ} \mathrm{C}$ en dos horas, especialmente durante el encendido de los hornos. Además se observaron eventos con máximos de temperatura de hasta $520{ }^{\circ} \mathrm{C}$ para el horno de cal. Los datos técnicos de las pinturas empleadas indican que estas soportan hasta $560{ }^{\circ} \mathrm{C}$ en un caso y hasta $150{ }^{\circ} \mathrm{C}$ en el otro. Sin embargo, el sistema de pintura de mayor resistencia no fue aplicado en la zona que alcanza las 
mayores temperaturas y por tanto, teniendo en cuenta la posibilidad de picos de temperatura por encima de los valores promedio, se hace muy probable el deterioro del recubrimiento, que luego por efectos atmosféricos resulta en su falla completa.

Según los registros de planta las láminas de los ductos de aire generalmente son en acero A-36 de calibre $3 / 16(4,7 \mathrm{~mm})$. Valores promedio medidos en tres puntos de la planta arrojan valores de 1,8, 3,3 y 3,8 $\mathrm{mm}$, esto es, reducciones de espesor del orden de $62 \%$, $30 \%$ y $19 \%$, respectivamente. Además se observaron perforaciones tanto en las juntas y uniones soldadas como aleatoriamente en las superficies. Las contribuciones a este deterioro son tanto por corrosión atmosférica en la superficie externa como por corrosión a temperatura elevada en la superficie interna, siendo esta última la que más contribuye como se observó en los análisis de las secciones transversales.

Los resultados de los monitoreos de velocidad de deposición o concentración de contaminantes atmosféricos se presentan en la tabla 1 para tres puntos estudiados.

Tabla 1. Velocidad de deposición/concentración de contaminantes atmosféricos.

\begin{tabular}{|c|c|c|c|}
\hline Contaminante & $\begin{array}{c}\text { Micro- } \\
\text { clima 1 }\end{array}$ & $\begin{array}{c}\text { Micro- } \\
\text { clima 2 }\end{array}$ & $\begin{array}{c}\text { Micro- } \\
\text { clima 3 }\end{array}$ \\
\hline $\begin{array}{c}\text { Cloruros } \\
{\left[\mathbf{m g ~ m}^{2} \mathbf{d}^{-1}\right]}\end{array}$ & 4,5 & 9,7 & 15,1 \\
\hline $\begin{array}{c}\text { Sulfatos } \\
{\left[\mathbf{m g ~ m}^{\mathbf{2}} \mathbf{d}^{-1}\right]}\end{array}$ & 7,2 & 17,8 & 31,1 \\
\hline $\begin{array}{c}\mathbf{N i t r i t o s}^{\text {Nito }} \\
{\left[\mu \mathbf{g ~ m}^{-3} \mathbf{N O}_{\mathbf{2}}\right]}\end{array}$ & 18,3 & 16,2 & 38,7 \\
\hline
\end{tabular}

En la norma ISO 9223 se establecen clasificaciones de la agresividad de la atmósfera según su contenido de cloruros y de sulfatos. De acuerdo a esto se tiene que los tres microclimas se pueden clasificar en el tipo $S_{1}$ para cloruros y $\mathrm{P}_{0}-\mathrm{P}_{1}$ para sulfatos, esto es, en los rangos de baja agresividad. Ahora, empleando los datos de temperatura y humedad relativa registrados en la planta, se puede calcular el tiempo de humectación, es decir, el número de horas en un periodo específico donde la humedad relativa es mayor del $80 \%$ y la temperatura está por encima de $0{ }^{\circ} \mathrm{C}$. Los datos indican que desde aproximadamente las 00:00 hora hasta las 07:00 de la mañana siguiente, se registran tales condiciones y por tanto podemos calcular un tiempo de humectación 2555 horas/año, que siguiendo la clasificación de la norma ISO 9223 corresponde a un nivel de agresividad $\mathrm{T}_{4}$, lo cual aplicaría para los tres microclimas. Así, estos datos indican que para los tres sitios evaluados se tiene un grado de corrosividad similar, establecido por la norma ISO como C3, nivel medio de corrosividad; para esta categoría se estima teóricamente una velocidad de corrosión en acero al carbono de 25 a 50 micrómetros por año.

Ahora bien, aun cuando el efecto de los óxidos de nitrógeno no ha sido cuantificado, se ha sugerido que la concentración mínima para llegar a incidir en la corrosión de los metales es del orden de $30 \mu \mathrm{g} \mathrm{m}^{-3}$ de $\mathrm{NO}_{2}$ [1], este nivel es solo sobrepasado en el Microclima 3 y por tanto sería el más agresivo. De otro lado se debe considerar el efecto del material depositado sobre las superficies el cual en general acelera los procesos corrosivos y para el caso de la planta es de importancia, formando depósitos compactos y adherentes que no son fácilmente lavados por las lluvias, como se pudo observar durante la inspección visual de la planta.

En cualquier caso los niveles mayores de contaminantes registrados en el Microclima 3 están sin duda relacionados con los efectos de su cercanía a un río. Finalmente, podemos decir que los niveles en los tres puntos son relativamente bajos y se asemejan mucho a un ambiente urbano moderado, lo cual no era de esperarse para una zona industrial, donde regularmente la presencia de contaminantes atmosféricos hacen más agresivo el ambiente.

En la tabla 2 se presentan los resultados del análisis de gases a la salida de la chimenea, los datos de presión parcial a $500{ }^{\circ} \mathrm{C}$ fueron calculados teóricamente. La concentración de $\mathrm{HCl}$ medida indica que en este gas no se favorece la formación de especies de cloruro que podrían acelerar la corrosión del metal [5, 9]. En pruebas de corrosión sobre acero al carbono A210, con concentraciones del orden de 100 ppm volumétricas de $\mathrm{HCl}$, se reporta un incremento en la velocidad de corrosión con la temperatura; al alcanzar $570{ }^{\circ} \mathrm{C}$ el $\mathrm{FeCl}_{2}$ formado sobre la superficie se puede volatilizar, generando huecos considerables debajo de la capa de óxido [5]. En otro estudio realizado sobre muestras de hierro altamente puro $(99 \%)$ y empleando una mezcla de gases con $\mathrm{HCl}$ en 4 x $10^{-4} \%$ (4 ppm), agua $6.1 \%$ y nitrógeno (balance) a una temperatura de $400^{\circ} \mathrm{C}$, se obtuvo una cinética parabólica, en la cual la corrosión inicia rápido y se estabiliza con el tiempo, formando además capas de óxido muy compactas sobre la superficie [9]. $\mathrm{El} \mathrm{SO}_{2}$ por sí solo aumenta la pérdida de metal en ambientes a alta temperatura [10] y cuando se encuentra en presencia de $\mathrm{HCl}$ en dichos ambientes el efecto agresivo se incrementa aún más [5]. Bajo sucesivas exposiciones a estos ambientes, la herrumbre se vuelve más porosa, frágil y poco adherente, quedando expuesto el material metálico y continuando 
así su ataque [5]. Ahora bien, se ha establecido que una relación de $\mathrm{SO}_{2} / \mathrm{HCl}$ por encima de 2 en la atmósfera puede favorecer la conversión de cloruros alcalinos a sulfatos alcalinos en el depósito, lo cual lleva a minimizar el ataque del cloro [5]; en el presente caso esta relación es de 3,27 y por tanto se espera una disminución en la corrosión por efecto de especies con cloro.

Para el análisis del efecto de las especies de azufre, cloro y carbono sobre la corrosión del metal se emplearon diagramas de estabilidad termodinámica de fases. Para estudiar el efecto del azufre se empleó el correspondiente diagrama de Ellingham [11] y los valores de las presiones parciales de las especies involucradas presentadas en la tabla 2. Así, teniendo en cuenta el equilibrio $2 \mathrm{Fe}+\mathrm{S}_{2}$ $\Leftrightarrow 2 \mathrm{FeS}$, del diagrama de Ellingham se puede estimar una presión parcial para el $\mathrm{S}_{2}$ a $200{ }^{\circ} \mathrm{C}$ de alrededor de $5 \times 10^{-28}$ atm. la cual es bastante menor que el valor obtenido de las medidas en la chimenea; esto indica que bajo las condiciones del gas de chimenea se favorece la formación y estabilidad del FeS. Para una temperatura de $500{ }^{\circ} \mathrm{C}$ se logran similares resultados al obtenerse del diagrama de estabilidad una presión parcial para el $\mathrm{S}_{2}$ de aproximadamente $5 \times 10^{-18} \mathrm{~atm}$.

Tabla 2. Resultados del análisis de gases de chimenea.

\begin{tabular}{|c|c|c|c|}
\hline $\begin{array}{c}\text { Especie } \\
\text { gaseosa }\end{array}$ & $\begin{array}{c}\text { Concentración } \\
{[\%]}\end{array}$ & $\begin{array}{c}\text { Presión } \\
\text { parcial } \\
\mathbf{a} \text { 200 } \\
\text { [atm] }\end{array}$ & $\begin{array}{c}\text { Presión } \\
\text { parcial } \\
\mathbf{a} 500 \\
\text { [atm] }\end{array}$ \\
\hline $\mathrm{CO}_{2}$ & 10 & 0,10 & 0,14 \\
\hline $\mathrm{SO}_{2}$ & $76 \times 10^{-4}$ & $7,5 \times 10^{-5}$ & $1,0 \times 10^{-4}$ \\
\hline $\mathrm{NO}$ & $766 \times 10^{-4}$ & $7,6 \times 10^{-4}$ & $1,0 \times 10^{-3}$ \\
\hline $\mathrm{N}_{2} \mathrm{O}$ & $1,0 \times 10^{-4}$ & $1,4 \times 10^{-6}$ & $2,0 \times 10^{-6}$ \\
\hline $\mathrm{HCl}^{\circ}$ & $23 \times 10^{-4}$ & $2,3 \times 10^{-5}$ & $3,1 \times 10^{-5}$ \\
\hline $\mathrm{O}_{2}$ & 2,0 & 0,02 & 0,03 \\
\hline $\mathrm{CO}$ & $10 \times 10^{-4}$ & $9,7 \times 10^{-6}$ & $1,3 \times 10^{-5}$ \\
\hline $\mathrm{N}_{2}$ & 61 & 0,61 & 0,83 \\
\hline $\mathrm{S}_{2}{ }^{*}$ & $3,8 \times 10^{-3}$ & $3,7 \times 10^{-5}$ & $5,1 \times 10^{-5}$ \\
\hline $\mathrm{Cl}_{2}{ }^{*}$ & $1,1 \times 10^{-3}$ & $1,1 \times 10^{-5}$ & $1,5 \times 10^{-5}$ \\
\hline $\mathrm{Humedad}^{2}$ & 27 & 0,27 & 0,36 \\
\hline
\end{tabular}

*Estos datos resultan de cálculos teóricos basados en los equilibrios: $2 \mathrm{SO}_{2} \Leftrightarrow \mathrm{S}_{2}+2 \mathrm{O}_{2}$ y $2 \mathrm{HCl}+1 / 2 \mathrm{O}_{2} \Leftrightarrow \mathrm{Cl}_{2}+\mathrm{H}_{2} \mathrm{O}$.

Ahora bien, considerando el equilibrio $2 \mathrm{FeS}+\mathrm{S}_{2} \Leftrightarrow$ $2 \mathrm{FeS}_{2}$, la presión obtenida del diagrama para el $\mathrm{S}_{2}$ a una temperatura de $500{ }^{\circ} \mathrm{C}$ es de $3 \times 10^{-5}$ atm., que en este caso es menor que la obtenida de medidas experimentales y por tanto a esta temperatura la fase estable será el $\mathrm{FeS}_{2}$; a $200{ }^{\circ} \mathrm{C}$ la anterior reacción no es posible y por tanto la fase estable será el FeS. La presencia de azufre en los productos de corrosión de la superficie metálica al interior del ducto fue confirmada por los análisis EDS.

De otro lado, empleando el diagrama de estabilidad Fe-O-S para $500{ }^{\circ} \mathrm{C}[10]$, con los datos de presión parcial de $\mathrm{S}_{2} \mathrm{y}$ $\mathrm{O}_{2}$ de la tabla 2, se tiene que la fase termodinámicamente estable a estas condiciones es $\mathrm{Fe}_{2}\left(\mathrm{SO}_{4}\right)_{3}$ sobre FeS y FeS . Esto es soportado por los análisis de FTIR de productos recolectados en superficies internas, los cuales indicaron la presencia de un compuesto tipo sulfato de hierro.

Para analizar el efecto de los compuestos de cloro se realiza un planteamiento similar, empleando el diagrama de estabilidad $\mathrm{Fe}-\mathrm{Cl}-\mathrm{O}$ a $500{ }^{\circ} \mathrm{C}$ [12] y considerando las presiones parciales de $\mathrm{Cl}_{2} \mathrm{y} \mathrm{O}_{2}$ de la tabla 2. De dicho diagrama para estas condiciones se tiene que las fases termodinámicamente estables a esa temperatura son las especies oxidadas del hierro tipo $\mathrm{Fe}_{2} \mathrm{O}_{3}$; lo cual está de acuerdo con lo encontrado en los análisis de la herrumbre realizados por DRX y FTIR.

Finalmente el potencial de carburización del metal se puede calcular a partir de las presiones parciales del $\mathrm{CO}$, $\mathrm{CO}_{2}$ y $_{2}$ y el diagrama de estabilidad Fe-C-O [13]. A partir de los datos de la tabla 2 para una temperatura de $500{ }^{\circ} \mathrm{C}$, se encuentra que a esas condiciones el potencial de carburización es muy bajo y la fase más estable es el $\mathrm{Fe}_{2} \mathrm{O}_{3}$, en concordancia con los análisis FTIR y DRX.

\section{CONCLUSIONES}

Según los resultados obtenidos se puede establecer que el problema de corrosión en las superficies externas es medianamente agresivo o nivel C3 según la clasificación de la norma ISO 9223. En este proceso influyen sinérgicamente la alta temperatura de los ductos, el prolongado tiempo de humectación, el material particulado, errores en la selección del sistema de protección con pintura y de manera particular las estructuras cercanas al río pueden verse más afectadas. La capa de productos formada es muy uniforme y compacta, estando constituida principalmente por hematita.

El deterioro en la superficie interna avanza por la ocurrencia de procesos de sulfurización y oxidación principalmente. La sulfurización es mayormente causada por el azufre liberado durante la combustión del carbón e induce un ataque de 
tipo localizado, el cual puede generar perforación de la pared metálica. Los resultados de los análisis indican la formación de sulfatos de hierro. Asimismo por reacción con la calcita de la materia prima se puede producir sulfato de calcio, el cual forma depósitos que incrementan la agresividad del medio al disminuir la presión parcial de oxígeno e incrementar aquella del azufre. Los productos de Fe-S son poco adherentes y por tanto poco protectores. De los procesos de oxidación el producto principal es el $\mathrm{Fe}_{2} \mathrm{O}_{3}$, común para las condiciones encontradas en este caso. Influencias de otros agentes pueden deberse a la formación de depósitos sobre la superficie por deposición de material particulado arrastrado por el gas.

Acerca del estado actual de la estructura se puede decir que existen grados de deterioro avanzados con pérdidas de espesor hasta del $62 \%$, en cuyos casos se requiere reemplazo de la estructura o la parte afectada. De otro lado el potencial de la estructura indica que el proceso corrosivo se encuentra activo pero no se detectó la ocurrencia de corrientes foráneas que estén agravando el ataque corrosivo.

\section{REFERENCIAS}

[1] C. Arroyave. "El papel del $\mathrm{NO}_{\mathrm{x}}$ en la corrosión atmosférica del acero". Tesis para optar al grado de doctor. Universidad Complutense de Madrid, CENIM-CSCI. Madrid, España. 1995.

[2] H. Kodama. "Infrared Spectra of minerals. Reference guide to identification and characterization of minerals for the study of soil". Research Branch Agriculture of Canada. Ontario, Canada. 1985.

[3] R.A. Nyquist and R.O. Kagel. "Infrared spectra of inorganic compounds". Academic Press, New York, USA. 1971.

[4] U. Schwertmann. "Iron Oxides in the laboratory". VCH. Weinheim. Alemania. 1991.

[5] X. Peng, K. Liu, W.P. Pan and J.T. Riley. "Hightemperature corrosion of A210-C carbon steel in simulated coal-combustion atmospheres". Oxidation of metals. Vol. 60, No 1-2, pp. 117-135. August 2003.

[6] M. Morcillo. "Soluble salts: their effect on premature degradation of anticorrosive paints". Progress in Organic Coatings. Vol. 36, pp. 137-147. 1999.

[7] J.M. Costa. "Fundamentos de electrónica, cinética electroquímica y sus aplicaciones". Ed. Alambra. Madrid, España, p. 367. 1981.

[8] M. Pourbaux. "Atlas of electrochemical equilibria in aqueous solutions". NACE. Houston. USA. 1974.

[9] Y. Sato and D.J. Young. "High-Temperature corrosion of iron at $900{ }^{\circ} \mathrm{C}$ in atmospheres containing $\mathrm{HCl}$ and H2O”. Oxidation of Metals. Vol. $55 \mathrm{~N}^{\circ} 3-4$, pp. 243-260. April. 2001.

[10] A. Järdnäs, J.-E. Svensson and L.-G. Johansson. "The inhibitive effects of traces of $\mathrm{SO}_{2}$ on the oxidation of iron". Oxidation of Metals. Vol. 60 No 5-6, pp. 427-445. December 2003.

[11] F.J. Bolívar. "Evaluación del comportamiento a elevadas temperaturas de recubrimientos $\mathrm{Al}$, Si y de Al modificado con Si y Hf depositados mediante CVD-FBR sobre aceros ferrítico martensíticos". Tesis para optar al grado de doctor. Universidad Complutense de Madrid. Madrid, España, p. 58. 2007.

[12] K. Zhang, Y. Niu, M. Al-Omary and W. T. Wu, "The corrosion behavior of four commercial steel in reducing atmospheres containing $\mathrm{HCl}$ at 773-873 K". Oxidation of Metals. Vol. 62 No 5-6, pp. 323-340. December 2004.

[13] G.Y. Lai. "High Temperature corrosion of engineering alloys". Haynes International Inc. Kokomo Indiana. USA, p. 50. 1990. 